\title{
Lymphatic drainage function and its immunological implications: From dendritic cell homing to vaccine design
}

\author{
Melody A. Swartz*, Jeffrey A. Hubbell, Sai T. Reddy \\ Institute of Bioengineering, École Polytechnique Fédérale de Lausanne (EPFL), Lausanne, Switzerland
}

\begin{abstract}
The slow interstitial flow that drains fluid from the blood capillaries into the lymphatic capillaries provides transport of macromolecular nutrients to cells in the interstitium. We discuss herein how this flow also provides continuous access to immune cells residing in the lymph nodes of antigens from self or from pathogens residing in the interstitium. We also address mechanisms by which dendritic cells in the periphery sense interstitial flow to home efficiently into the lymphatics after activation, and how lymphatic endothelium can be activated by this flow, including how it can act as a lymphatic morphoregulator. Further, we present concepts on how interstitial flow can be exploited with biomaterial systems to deliver antigen and adjuvant molecules directly into the lymphatics, to target dendritic cells residing in the lymph nodes rather than in the peripheral tissues, using particles that are small enough to be carried along by flow through the network structure of the interstitium. Finally, we present recent work on lymphatic and lymphoid tissue engineering, including how interstitial flow can be used as a design principle. Thus, an understanding of the physiological processes that govern transport in the interstitium guides new understanding of both immune cell interactions with the lymphatics as well as therapeutic interventions exploiting the lymphatics as a target.
\end{abstract}

(C) 2007 Elsevier Ltd. All rights reserved.

Keywords: Immunotherapy; Engineering; Lymph node; Biomaterials; Lymphatic morphogenesis; Adjuvant; Complement; Chemotaxis; Flow

Dendritic cells (DCs) are the most potent and important antigen-presenting cells (APCs) because of their capacity to take up and process antigens and present them to naïve T cells $[1,2]$. They also can fine-tune immune responses by instructing $\mathrm{T}$ cell differentiation and polarization (i.e., they can induce humoral, cellular (cytolytic), or suppressive responses in T cells). In addition to these important roles in biomolecular recognition and signaling, DCs are physically positioned to take up antigen from peripheral tissues and carry them to the lymph node so that circulating $\mathrm{T}$ cells only need to traffic through the lymph nodes to interact with all APCs. The lymphatic vessels are thus critical for transporting activated DCs from the periphery to the lymph node. They constantly deliver a representative sampling of interstitial fluid to the lymph node, allowing immature DCs and macrophages there to screen potential antigens and also to carry inflammatory signals from the tissue (e.g., cytokines, extracellular matrix fragments, etc.) to the lymph node faster than peripheral, migrating DCs can, possibly preparing the lymph

\footnotetext{
* Corresponding author at: Institute of Bioengineering, SV/IBI/LMBM, Station 15, École Polytechnique Fédérale de Lausanne (EPFL), CH-1015 Lausanne, Switzerland. Tel.: +41 21693 9686; fax: +41216931660.

E-mail address: melody.swartz@epfl.ch (M.A. Swartz).
}

node for the need to increase surveillance there in preparation for a pending immune response. Until recently, lymphatic vessels have been considered a passive conduit for immune cells. This review explores the links between lymphatic physiology and biology with trafficking of antigen and DCs from the interstitium of the peripheral tissues eventually into the lymph nodes, and discusses the implications of these links on immunomodulation and vaccination strategies.

\section{Interstitial flow and lymphatic drainage}

The lymphatic system complements the venous branch of the circulation by draining excess fluid and solutes from the interstitial space and returning them to the blood. In this way, lymphatic drainage facilitates low levels of interstitial flow from the blood capillaries to perfuse the tissue interstitium; without this interstitial flow, transport of proteins, macromolecules, viruses, nanoparticles, and other large solutes within the tissue interstitium would occur by diffusion alone, which can be exceedingly slow (diffusion coefficients on the order of $10^{-7}$ to $10^{-8} \mathrm{~cm}^{2} / \mathrm{s}$ for typical proteins). Importantly, lymphatics are also a major route for cell transport, since lymphatic capillaries drain to collecting vessels and pass through lymph nodes 


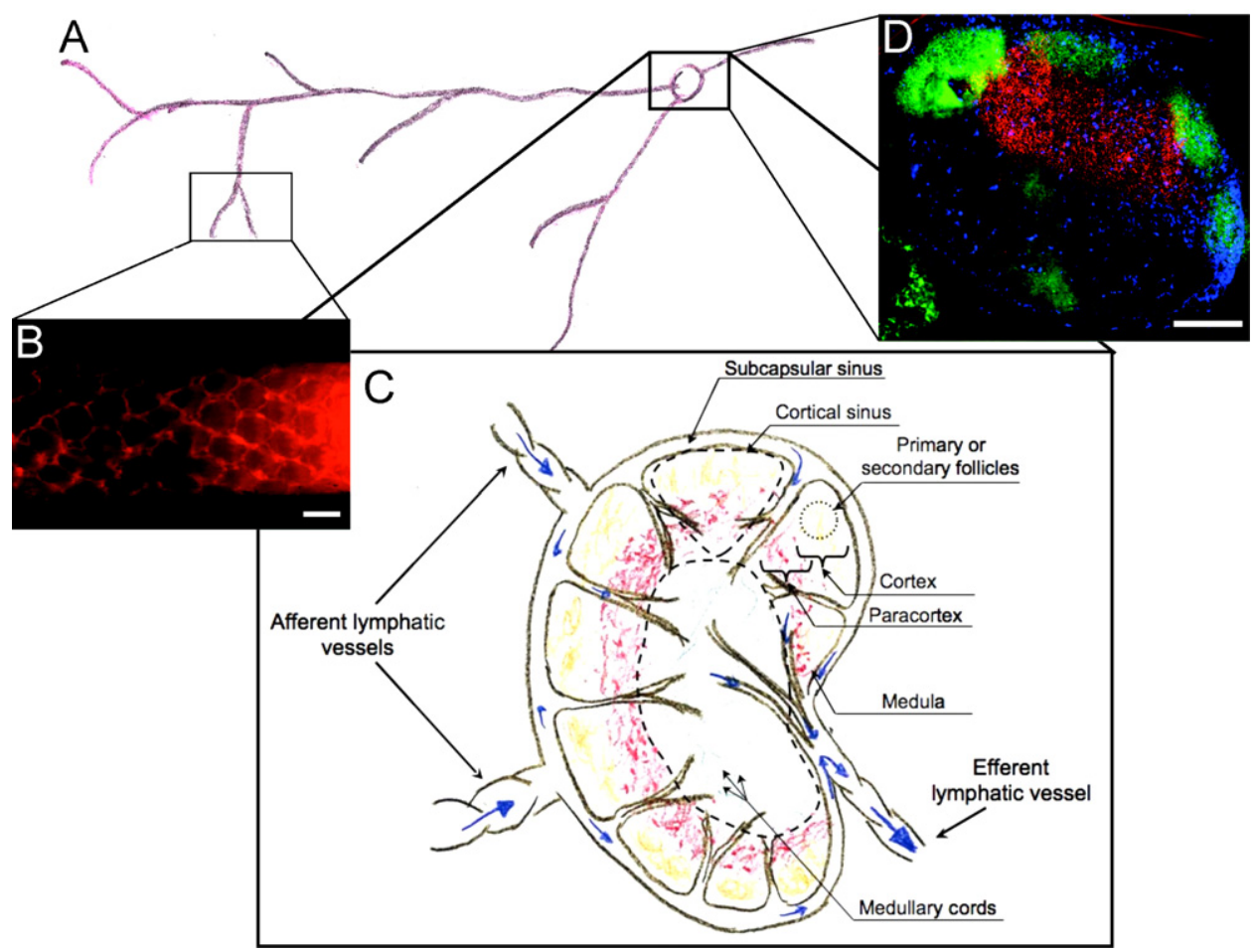

Fig. 1. Schematic of lymph flow. (A) Overall schematic showing lymphatic capillaries draining to collecting vessels and into lymph nodes. (B) Lymphatic capillaries are arranged in an interconnected mesh-like network of vessels, as demonstrated here by the regular hexagonal network found in the mouse tail skin made visible by the intradermal injection of a red fluorescent macromolecule $(\mathrm{bar}=100 \mu \mathrm{m}) .(\mathrm{C})$ The major regions of the lymph node include the cortex, paracortex, and medulla, and cortical nodules are divided by connective tissue called trabeculae. The cortex contains the primary cortical follicles that become secondary follicles with germinal centers upon antigenic stimulation; the cortex is the primary site of B cell residence, antigen presentation, and antibody production. The paracortex surrounds the B cell follicles and is the main site for T cell homing. DCs can be found throughout the node but are in highest numbers in the paracortex and medulla. Lymph enters through afferent lymphatic vessels (that contain valves to prevent backflow), around the subcapsular sinus and into the node through a network of reticular fibers, and exit via the efferent lymphatic vessel. Large molecular weight particles may remain in the subcapsular sinus and bypass the node. (D) Section of a mouse lymph node stained for B cells $\left(C D 45 \mathrm{R}^{+}\right.$cells, green), $\mathrm{T}$ cells $\left(\mathrm{CD} 3 \mathrm{e}^{+}\right.$cells, red), and antigen-presenting cells $\left(\mathrm{CD} 68^{+}\right.$macrophages and $\mathrm{DCs}$, blue $) . \mathrm{Bar}=100 \mu \mathrm{m}$.

before emptying into the blood via lymphatic ducts. Indeed, lymph nodes (along with other secondary lymphoid organs such as the spleen) are the most important sites for immune cells to exchange information, reside, expand, and initiate adaptive immune responses. By draining interstitial fluid and carrying any potential antigens to lymph nodes, the lymphatic system optimizes immune response because it allows circulating antigen-specific lymphocytes to patrol all the lymph nodes for their cognate antigens rather than having to sample peripheral tissues directly. As such, one might also think of lymphatic vessels as "sensory" organs of the immune system, and of their drainage function primarily as a means to sample peripheral tissue fluid and all antigens contained there (Fig. 1).

The lymphatic endothelium itself optimizes this transport role: it has discontinuous basement membrane and weak cell-cell junctions, making it highly permeable to fluid and high molecular weight solutes such as proteins and even nanoscale colloidal particles. Lymphatic capillaries vary in size and are not circular in cross-section, and their diameters range from 10 to $80 \mu \mathrm{m}$ [3-7]. They drain fluid by virtue of their anchoring filaments, which pull the lymphatic lumen open during slight changes in tissue hydration, causing a subsequent pressure drop that drives fluid flow into the lymphatic lumen [8]. Backflow out of the vessel is prevented by these overlapping cell-cell junctions that act like valves $[3,4,9]$ : as the lymphatic capillary fills, they close and prevent backflow. Thus, lymphatic function causes interstitial fluid flow that is always directed towards the draining lymphatic capillary.

Once lymph is formed into the lymphatic capillaries, it flows to deeper collecting lymphatic vessels, which are segmented into lymphangions separated by valves and innervated by surrounding smooth muscle cells that have intrinsic contractility with a magnitude range similar to that of cardiac muscle. Their behavior depends on pressure and flow conditions [10,11]: when transporting lymph up a pressure gradient, they contract and pump fluid from one lymphangion to the next in a wave propagation manner dependent on gap junctional communication [12], and when transporting lymph down a pressure gradient (high load conditions), they can act as conduits with decreased contraction amplitude, modulating tone and regulating flow resistance. These prenodal collecting vessels are also referred to as afferent lymphatic vessels and lead to lymph nodes, allowing the contents of lymph to be sampled by immune cells as well as transporting activated dendritic cells there. In the human, there are 450 lymph nodes ( 22 in the mouse) and they are generally $1-2 \mathrm{~cm}$ in diameter $(0.5-2 \mathrm{~mm}$ in mouse). Lymph can pass through several different lymph nodes before being returned to the blood via the thoracic duct. 


\section{Flow through the lymph node}

From the perspective of lymph flow, the lymph node architecture should be adapted in such a way that antigen-presenting cells are exposed to any antigens or pathogenic material picked up by the lymph from the peripheral tissues, and that immune cell trafficking is optimized to allow the antigen-specific $\mathrm{T}$ cell to find its cognate antigen-presenting DC (Fig. 1C). Lymph flows through the afferent vessels into the subcapsular sinus, through capsular sinusoids and into the medullary sinusoids before leaving the lymph node via efferent vessels. It can also flow through fine reticular fibers that extend into the $T$ cell regions $[13,14]$ and where immature DCs, but not immigrated mature DCs, appear to also reside [15]. This reticular network consists of collagen fibers, basement membrane components, and reticular fibroblastic cells [13], and is believed to further guide lymph flow and T cell-DC interactions there [14]. Blood flows through the high endothelial venules (HEVs) that have loose cell-cell junctions and contain adhesion receptors like intercellular adhesion molecule (ICAM)-1 and L-selectin to facilitate exit of antigen-specific lymphocytes from the blood into the lymph node in search of cognate antigen. Furthermore, activated (antigen-presenting) DCs migrate near these HEVs to optimize their chances of finding the rare antigen-specific naïve $T$ cell partner. Cell migration and communication among immune cells within the lymph node is complex and involves at least several chemokine and sphingosine-1-phosphate (S1P) receptors [16] whose ligands are established by stromal cells as well as immune cells themselves [17-20]. Cell-cell communication and probing is also enabled by the long dendritic extensions on DCs, which allow them to sample a much greater space, and therefore contact more $\mathrm{T}$ cells, than would be possible otherwise [21].

During inflammation, tissue fluid drainage can be increased 10 -fold or more $[6,22,23]$, which increases both the rate of antigen delivery from the periphery to the lymph node as well as DC trafficking (which will be explained below). Furthermore, cytokines that are produced in the affected site are carried to the draining lymph node, where they can induce changes in the lymph node that enable and enhance immune cell trafficking there, including the expansion of the feed arteriole which increases blood flow and therefore lymphocyte trafficking to the affected node. For example, the inflammatory cytokines IL-6 and IL-8 can increase the expression of lymphocyte receptors like ICAM-1 and the homing chemokine CCL21 by HEVs [24]. Inflammation also apparently can induce expansion of the lymphatic network in the lymph node $[1,25]$, which may help recruit more DCs from the periphery into the lymph node or enhance T cell trafficking out of the node. Fever can also enhance ICAM1, L-selectin, and CCL21 expression by HEVs. Thus, a number of positive feedback mechanisms exist by which inflammation induces enhanced exposure of peripheral antigens to the lymph nodes.

When lymphatic vessels are blocked or compromised in their capacity to drain fluid, lymphedema can occur. This is associated with tissue fluid stagnation, enlargement of the limb, accumulation of lipids, and impaired immune function [26].
Very little is known about how immune cell trafficking and the immune response is altered in lymphatic dysfunction, but, given the importance of lymphatic drainage to antigen delivery to the lymph node, and furthermore the importance of such interstitial flow on the physiology of DC homing to the lymphatics (see next section), the limitations in the immune response must be substantial. Hopefully more research in this area will help elucidate this topic in the near future.

\section{Implications of interstitial flow on DC homing to lymphatics}

One implication of interstitial flow from the blood capillaries to the lymphatics may be the facilitation of DC homing towards and into lymphatic capillaries, the first step in adaptive immunity, for example with Langerhans cells [2]. During these initial steps, DCs undergo a maturation process, which is mediated by the inflammatory signals and characterized by altered chemokine receptor expression including the downregulation of CCR1 and CCR5 (which bind CCL3 and RANTES) and the upregulation of CCR4 (which facilitates DC-T cell interactions in the lymph node) and, importantly, CCR7, which is necessary for homing towards lymphatic capillaries [27-29] (Fig. 2). Their transmigration into lymphatic vessels is not well understood, as is their entry into the lymph node from the afferent vessels, although both are critical for the immune response. Both known CCR7 ligands, namely CCL21 (SLC or 6-C-kine) and CCL19 (ELC or MIP-3ß), appear to be important in DC homing [30-32]. It is generally assumed that DC trafficking towards lymphatic vessels is primarily driven by chemotactic gradients of lymphatic-secreted CCL21, and that lymphatic capillaries broadcast this signal to attract DCs patrolling the interstitium.

However, this concept is confounded by two observations, indicating that there may be more subtle complexities to the story. First, the lymphatic capillary must broadcast CCL21 "upstream", i.e. against the draining flow into the capillary, before it can reach DCs in the interstitium. Given that the interstitial flow is on the order of magnitude of $0.1-1.0 \mu \mathrm{m} / \mathrm{s}$ [33], this represents a potentially important factor in to overall CCL21 transport. Second, CCL21 is strongly matrix-binding [34], which may substantially limit its broadcast distance through the interstitium; because of this, it is sometimes used as a marker for lymphatic endothelium as it is mostly found on the basal surface of lymphatic capillaries [35]. This situation is further exaggerated during acute inflammation, when interstitial drainage rates can increase an order of magnitude or more [22,36-38], and when DC homing is greatest (i.e., when the CCL21 signal is most needed). Furthermore, it is also interesting to note that both DCs and LECs secrete CCL19, ${ }^{1}$ which competes with CCL21 for CCR7 ligation but unlike CCL21 it does not bind to sulfated proteoglycans in the matrix (and thus it exists only in soluble form). The exact mechanism of this CCR7 ligand in mod-

\footnotetext{
1 We and others have noted that cultured LECs secrete CCL19 (unpublished observations); lymphatic secretion of CCL19 in vivo has not been well-established.
} 
(A)

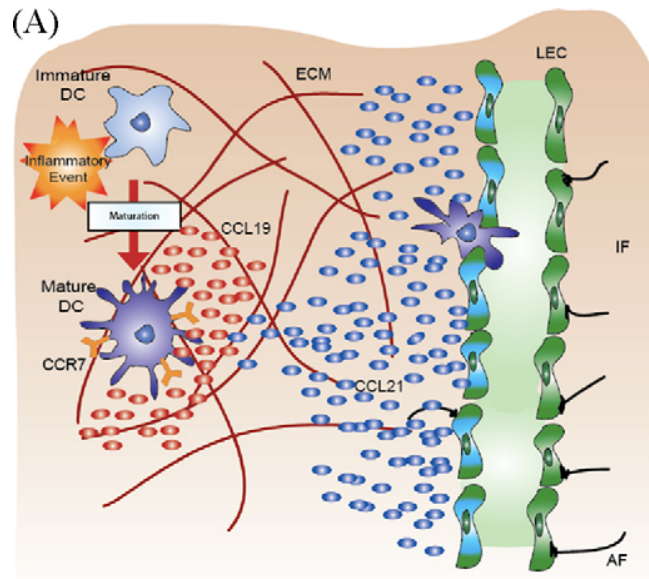

(B)

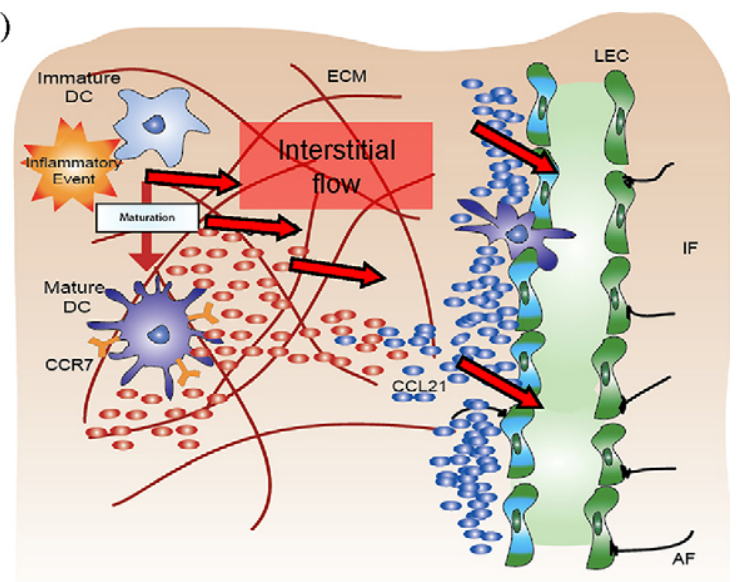

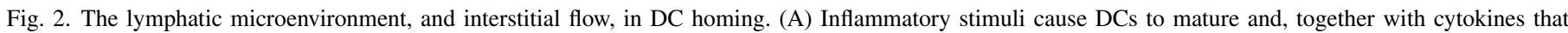

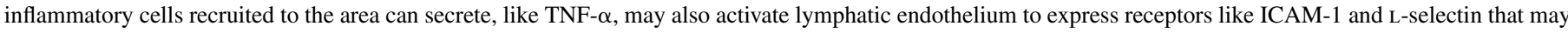

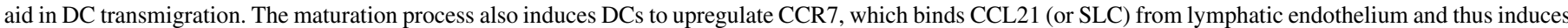

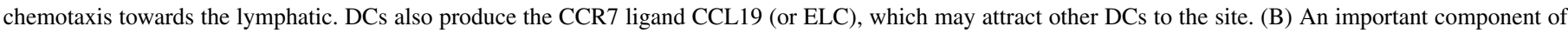

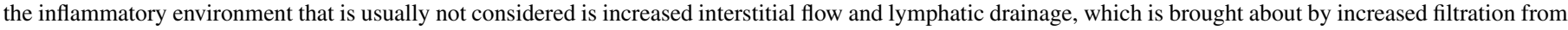

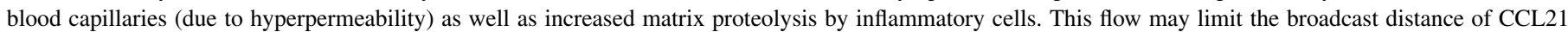

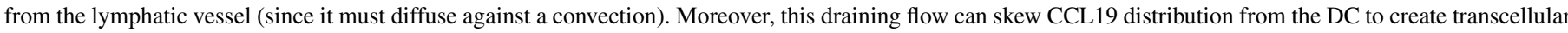

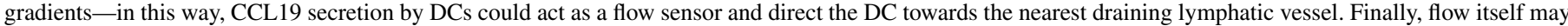
activate lymphatic endothelial cells to change permeability or increase chemokine secretion, for example. These roles need to be further elucidated.

ulating DC chemotaxis in the presence of CCL21 is unknown, although when CCL19 is blocked, DC migration to lymph nodes is reduced [32].

Furthermore, until very recently, the initial lymphatic vessel was considered a mostly passive conduit for DC trafficking [39], but new evidence suggests that the lymphatic vessels likely play a very active role. For example, it was recently shown that lymphatic endothelial adhesion receptor profiles change after exposure to inflammatory cytokines [40], although it is not known whether different molecules govern DC transmigration into lymphatics as opposed to leukocyte transmigration out of blood capillaries. Also, lymphatic endothelial cells have been shown to express Toll-like receptor (TLR) 2 and 4 [41], indicating that lymphatics may sense and respond to "danger signals" in the periphery, perhaps to prepare themselves for eventual DC trafficking. Finally, it is likely that the physical microenvironment of lymphatics, namely slow interstitial flow through a 3D matrix, can upregulate chemokine secretion and adhesion molecule expression on lymphatic endothelium (Miteva and Swartz, unpublished observations), suggesting a role of interstitial flow in further guiding DCs to lymphatics.

\section{Exploiting interstitial flow to target antigen to lymph node vs. peripheral DCs}

For many years the conventional view was that immature DCs existed only in peripheral tissues and that DCs in secondary lymphoid organs were in a mature state, i.e., they could not take up and process new antigen there. Therefore, vaccine strategies have targeted peripheral DCs by delivering antigen (and adjuvant, as discussed further below) to peripheral DCs in the skin or muscle, which in turn migrate to and enter the lymphatic capillaries and subsequently migrate to the lymph nodes to present antigen and co-stimulatory molecules to T cells. However, recent work has demonstrated that such a generalized view of DC phenotypes is inaccurate. A comprehensive study by Wilson et al. demonstrated indeed that the majority of DCs in lymph nodes (and spleen) were phenotypically and functionally immature [42]. These immature DCs in lymph nodes were found to be constantly sampling self-antigen and presenting to $\mathrm{T}$ cells for the purpose of maintaining tolerance; however when lymph node DCs were exposed to antigen simultaneously with a maturation stimulus, they were able to activate $\mathrm{T}$ cells, suggesting that they are fully capable of triggering immune recognition while resident within the lymph node and taking up antigen within the lymph node. The high concentration of immature DCs in lymph nodes compared to that in the peripheral tissues and their ability to process and present antigen makes lymph node-resident DCs an intriguing target for vaccine delivery, if co-delivery of antigen and adjuvant to the lymph node were achieved. As an additional benefit, there would be little risk of premature antigen presentation (when peripheral DCs become mature before reaching the lymph node), which could lead to tolerance rather than induction of adaptive immunity.

Exposure of DCs to antigen and activating molecules ex vivo has been explored to increase targeting efficiency by avoiding physiological barriers. Such ex vivo approaches have made considerable progress in research as well as clinical development; in such approaches, the DC is brought into direct contact with antigen and adjuvant ex vivo, obviating the need for any biomolecular or physiological targeting strategy. Such strategies consist of isolating monocyte precursors from patient blood, differentiating them into DCs with growth factor cocktails, exposing them to antigen and maturation stimuli, and finally re-injecting them back into patients [43-45]. One example of an ex vivo autologous DC vaccine is the therapeutic vaccine 
APC8015 (Provenge; Dendreon Corp, WA) to treat metastatic prostate cancer. In this strategy, isolated DCs are exposed to a recombinant fusion protein of GM-CSF attached to prostate specific antigen and then the DCs are injected into patients; a Phase III trial has shown prolonged survival in patients [46]. A similar strategy, where autologous DCs are pulsed with tumor cell lysate from surgically resected brain cancer tissue (DCVaxBrain, Northwest Biopharmaceuticals, WA) has also reached late-stage clinical trials [47]. Despite the promise of ex vivo DC vaccines, many logistical and economical complexities and therapeutic limitations remain. For example, autologous DC vaccines are not a realistic option for prophylactic regimes or treatment of infectious diseases in developing regions, where there is the greatest need for new vaccines. Therefore a strong interest exists to develop more robust vaccines with in vivo DC targeting.

Of course, targeting immature DCs residing in peripheral tissue such as skin are the most widely explored vaccine targeting approaches. Non-specific uptake of particles by DCs has been demonstrated with polymeric microparticles, virus-like particles, and polystyrene nanobeads [45,48-51]. However, the low concentration of DCs in peripheral skin has motivated strategies for more specific targeting through molecular recognition. Steinman and co-workers have designed a system to graft protein antigen to an antibody for the DC surface endocytosis receptor DEC205; they have demonstrated, with the addition of a molecular danger signal adjuvant, the ability to induce potent adaptive immune responses following peripheral administration [52-54]. A particulate approach has also been utilized for DC targeting by conjugating anti-DEC205 and anti-CD11c to microparticles and liposomes, respectively [55,56]. While molecular targeting of DCs has shown potential, there are still limitations in physiological transport (mentioned below), and economic feasibility that must be considered as well.

As an alternative to the ex vivo manipulation and biomolecular approaches mentioned above, we point out the basis for a biophysical approach, namely that the interstitial flow that drains fluid from the blood capillaries into the lymphatic capillaries can be exploited to deliver antigen to the lymphatics and thus to the lymph node-resident DCs. A major challenge to exploiting this flow is presented by the resistance to transport due to the network structure of the interstitium [57,58]. The interstitial space possesses a complex microarchitecture comprising fibrillar proteins and proteoglycans and offers a high resistance to molecular transport through the interstitium. Several investigators have demonstrated that the size of molecular solutes and nanoparticles influences their transport properties through the interstitium. One study quantified convective interstitial transport of injected macromolecules and colloidal nanoparticles through the interstitium in mouse skin and showed that solutes of 7-12 nm diameter moved optimally through the interstitium and into the lymphatics [59], and for nanoparticles, smaller was always better with 20-25 nm particles being taken up into the lymphatics from the interstitial space much more readily than $100 \mathrm{~nm}$ particles [60]. Liposome transport in the interstitium and subsequent uptake by the lymphatic capillaries and delivery to the lymph node has also been demonstrated to strongly depend on size, with $70 \mathrm{~nm}$ appearing as a cutoff for interstitial liposomal transport [61]. It is noteworthy that the size cutoff for transport through the interstitium seems to be more stringent than size cutoff for entry into lymphatic capillaries through their valve structure, with the interstitial resistance offering the major barrier [62]. Thus, it is apparent that interstitial flow may be used to direct nanoparticles to the lymph nodes, were DCs reside in high numbers, so long as the nanoparticles are sufficiently small to readily move through the tissue interstitium.

From the perspective of lymph node targeting, it is unclear if smaller is always better-while smaller particles may enter lymphatics more effectively, they also tend to be cleared through the lymph node more rapidly, partly because lymph node-resident macrophages and DCs can take up larger particles more efficiently than smaller ones [63]. It is not known if this same observation would be true with particles bearing ligands for macrophage and DC endocytosis receptors. Thus, from the perspective of efficient interstitial transport and prolonged lymph node retention, a delicate balance may exist and may require optimization for antigen delivery and retention to the lymph nodes via interstitial flow. The extent of this balance requires further elucidation, also in view of the unknown merits of prolonged retention within the first lymph node draining the injection site vs. within several nodes along the lymphatic chain.

\section{Exploiting interstitial flow to simplify adjuvant and vaccine design}

Because of the complexities of using attenuated pathogens, along with recent advances in recombinant DNA technology, vaccine technology approaches have shifted towards subunit and genetic vaccines that use protein, peptide, and DNA-encoded antigen. To date, challenges exist in these types of antigens being generally weak immunogens, and therefore considerable attention is devoted to developing new molecular adjuvants, broadly defined as compounds that enhance the immunogenicity of antigens.

Most adjuvants have been developed to function in the periphery. For example, the most widely used adjuvants are based on aluminum salts (alum). Despite nearly 60 years of clinical use, a complete mechanistic description of alum's mode of action remains elusive. Alum is thought to work by producing a depot effect and precipitating antigen so that it can be taken up more effectively by peripheral DCs [64]. In addition, alum induces non-specific inflammation, which helps recruit more DCs to the site of the injection depot. While alum is safe, it is also considered relatively weak and only capable of inducing $\mathrm{Th} 2$ immunity [65]. Therefore, a great deal of research exists to develop advanced adjuvants that are capable of inducing Th1 cell-mediated immune responses, which could provide better protection against viral and intracellular pathogens.

While alum was largely discovered through empirical methods, the recent explosion of knowledge in innate immunity has allowed new adjuvant research to be driven by more rational approaches. By far the most research has focused on designing molecular danger signal adjuvants targeting TLRs [66]. Pattern recognition receptors like TLRs are activated by 
"pathogen associated molecular patterns" (PAMPs). They are present on the surface and within the endosomes of many different immune cells, most notably DCs and macrophages. The primary outcome of TLR activation on DCs is maturation (upregulation of co-stimulatory molecules) and secretion of inflammatory cytokines which often leads to strong Th1 responses; therefore TLRs are excellent targets for vaccine adjuvants [67]. There have been at least 11 mammalian TLRs discovered to be activated by pathogenic signals such as bacterial lipopolysaccharide (LPS), fungal zymosan, viral singleand double-stranded RNA, and CpG containing DNA [68]. Synthetic derivatives of TLR activators are currently being exploited in experimental vaccine formulations. Monophosphoryl lipid A, a chemically modified derivative of LPS, signals through TLR4 and has seen significant clinical success in several vaccine formulations, including a vaccine for hepatitis B [66,69]. Other synthetic TLR agonists include unmethylated CpG DNA, which mimics a bacterial and viral DNA sequence that activates TLR9 [70]; polyinosinic:polycyidylic acid (poly I:C), a synthetic double-stranded RNA that activates TLR3 [71]; synthetic lipopeptide tripalmitoyl-S-glyceryl cystein (Pam3Cys), which activates TLR2; and imidazoquinolines, which are small molecule activators of TLR7/8 [70]. These TLR agonists are being explored in several experimental vaccines. In most if not all cases, they induce substantial expression and release of inflammatory cytokines (which in turn recruit DCs to the injection site and contribute to their maturation); as such, there is a delicate balance between efficacy and toxicity to be considered.

Co-administration of the antigen and adjuvant in a single nano- or microparticle, which may also provide prolonged presence of the adjuvant, is a promising approach. Monophosphoryl lipid A can be administered in soluble form or even precipitated with alum [72], but as elucidated above this makes it only able to target peripheral DCs, being excluded from the lymphatics by size. Co-loading of antigen and adjuvants into microparticles and liposomes has also been investigated, however the size of these carriers limits targeting to peripheral DCs at the site of injection. In a study by Wang et al., microspheres composed of poly(ortho ester) were designed to carry antigen-encoding DNA, protecting it from degradation and facilitating uptake into antigen-presenting cells [51]. Similar cancer vaccines based on gene delivery with microspheres made of poly( $\beta$-amino ester) have also been demonstrated in vivo $[49,73]$. Another microparticle approach has explored targeting DCs with anti-CD11c antibody, grafted to acid-degradable microparticles carrying protein antigen [55] and achieved strong cellular immunity when co-loaded with $\mathrm{CpG}$ oligonucleotides as a TLR9 activator [74]. Liposomes conjugated with DC-specific antibodies and co-loaded with antigen and molecular danger signals have also shown promising anti-tumor immunity [56].

Another major component of innate immunity is the complement system, a pathway of biochemical defense consisting of a number of proteins and enzymes that converge to opsonize pathogens and induce local inflammatory responses. Usually considered the first line of defense, complement activation was considered an effector arm of humoral (antibody) immunity; however, recent work has demonstrated the importance of complement in promoting adaptive immune responses as well. Adjuvants utilizing the complement system have been explored; one strategy developed a fusion of $\mathrm{C} 3 \mathrm{~b}$ or $\mathrm{C} 3 \mathrm{~d}$ to model antigens; following immunization in mice, a significant increase in the acquired immune B cell response was observed compared to free antigen alone [75]. The mechanism has been explained by the fact that $\mathrm{C} 3 \mathrm{~b}$ and $\mathrm{C} 3 \mathrm{~d}$ adjuvants work through direct binding of the C3d receptor (CD21/35), which associates with CD19, a known amplifier of $\mathrm{B}$ cell activation [76]. Complement has also been discovered to play a role in T cell-dependent immunity [77], and while a complete description of the molecular mechanisms responsible have yet to be described, it is likely that complement proteins affect the complex signaling interactions between DCs and $\mathrm{T}$ cells [78].

This newly discovered role of complement in T cell immunity suggests complement activation as a vaccine adjuvant. One approach to activating complement is with biomaterials, since it is well-known that many polymer surfaces can activate complement via the alternative pathway by reaction of hydroxyl groups on the biomaterial surface reacting with $\mathrm{C} 3$, leading to biomaterial-bound $\mathrm{C} 3 \mathrm{~b}[79,80]$. For example, biomaterial coatings with polyethylene glycol or its copolymers such as Pluronic, used in many particle based delivery systems, have been shown to activate complement $[81,82]$. The advances in molecular understanding of innate immunity seen in recent years with TLRs and complement have brought incredible interest to vaccine adjuvant research, as finally there is hope for a clinical alternative to alum.

Returning to interstitial flow, and how recognition of its function would enable re-consideration and perhaps simplification of adjuvant design, we have noted above the potential disadvantages of coupling the adjuvant to particles so large as to exclude transport through the interstitium. We also noted that, if the antigen and adjuvant are both sufficiently small to be convected into the lymphatics, there is no remaining need for an adjuvant to be designed to induce inflammation and thereby induce DC trafficking to the peripheral site. Thus, agents that induce a less potent response of cytokine expression and release may be considered, and, as such, a major source of toxicity might be avoided: it is not necessary for the adjuvant to induce inflammation to attract and recruit DCs to the site of injection, it need only activate the DCs once it encounters them in the node.

As one specific approach to integrate the above knowledge of DC activation and interstitial transport into the lymphatics, very small polymer particles bearing both antigen and complementactivating surface chemical features have been explored $[60,83]$. Using this approach, in situ complement activation served as the danger signal for DC maturation. Further, this activation and induction of antigen presentation by the DCs occurred within the lymph node, since the 20-25 nm nanoparticles employed were convected to the lymphatics within a few tens of minutes. Given the high concentration of DCs present within the lymph node, and due to the efficient transport of the very small nanoparticles into the lymphatics, it was possible to deliver the antigen and adjuvant (on the same nanoparticles) to more than $50 \%$ of the DCs present within the lymph node that drained the injection site - a remarkable level of efficient delivery. This approach was 
demonstrated to induce a strong humoral and cellular adaptive immune responses to antigen bound on the polymer nanoparticles, comparable to that achieved with LPS as the adjuvant, which is far too toxic to contemplate for clinical use. In spite of this strong immune stimulation, signs of local inflammation that would be of clinical consequence were absent [83].

\section{Lymphatic tissue engineering}

There is a need for lymphatic tissue engineering to generate mimetics of the lymph node for basic biological studies, to form lymph node equivalents for immunotherapy, and to generate lymphatic vessels for basic physiological studies as well as potentially for the treatment of lymphedema. Furthermore, lymphatic vessel tissue engineering in biopolymer matrices, synthetic matrices, or engineered tissues such as liver should be a central consideration in tissue engineering and regenerative medicine, since lymphatic vessels are critically important for both modulating proper immune surveillance of the implanted matrix or engineered tissue as well as maintaining hydrostatic and osmotic pressures there. At present, lymphatic tissue engineering is very much in its infancy, and much of the above remains conceptual, with only few foundational demonstrations of those concepts.

Initial progress in lymph node tissue engineering has explored developing model systems for studying the dynamic interactions between cells in the lymph node. Surface patterning methods have been employed to generate precise domains that bear molecular signals that are normally presented by APCs, such as $\mathrm{T}$ cell receptor ligands and ICAM-1, to more precisely understand the interactions between DCs as presenters of those signals and $\mathrm{T}$ cells as receivers and explore the regulation of synapse formation between them [84,85]; this is addressed elsewhere in this issue. With regard to generation of engineered lymph node environments for basic immunological investigation, porous materials have been explored as model microenvironments to explore the mechanisms by which DCs present ligands to a large number of $\mathrm{T}$ cells within the lymph node microenvironments [86].

Progress has also been made in formation of ectopic artificial lymph node-like tissues for immunotherapy using biomaterials and transplanted cells. Absorbable collagen sponges have been seeded with thymus-derived stromal cells that expressed lymphotoxin $\beta$ receptors and vascular cell adhesion molecule (VCAM)-1 with and without co-seeding with activated bone marrow-derived DCs. These constructs, when implanted in the renal subcapsular space, evolved over a 3-week period of time in vivo into lymph node-like tissues, displaying $\mathrm{B}$ cell and $\mathrm{T}$ cell zones, germinal centers, follicular DC networks and even high endothelial venules, indicative of appropriate connection of the artificial lymph node to at least the blood vasculature [87]. These artificial lymph nodes have been demonstrated to be competent to induce secondary immune responses in mouse models [88]. Initial results in generation of artificial lymph nodes are very exciting, however even more exciting would be implantation of cytokine-loaded biomaterial scaffolds to induce immigration of the appropriate immune, stromal and vascular cells to achieve lymph node-like structures, but this at present remains an unrealized concept.

Lymphatic vessel tissue engineering, again very important but largely underdeveloped for the general field of tissue engineering and regenerative medicine, can be considered in two general approaches: (1) developing lymphatic capillary networks in vitro (incorporated within the engineered tissue or throughout a synthetic or natural biopolymer matrix) that will later anastomose with existing lymphatics post-implantation, and (2) developing strategies to promote lymphangiogenesis into the implanted tissue or matrix-binding growth factors, or controlled release of growth factors from implanted matrices.

Both approaches are newly facilitated by the recent explosion of research in lymphangiogenesis biology, although much still needs to be learned about the functional (as opposed to developmental) biology of lymphatic endothelium. VEGF-C and VEGF-D, ligands of VEGFR-3, are the most well-studied lymphatic growth factors, and play similar roles for lymphangiogenesis to those of VEGF in blood angiogenesis; Prox-1 (which promotes differentiation of the lymphatic lineage from veins), Ang-2 (which is important in lymphatic patterning), VEGF, and many other factors have also been demonstrated to be critical for lymphangiogenesis (reviewed in $[89,90]$ ). Aside from growth factors, interstitial fluid flow has also been shown to play an important organizational role in lymphangiogenesis both in vivo $[35,91,92]$ and in vitro $[93,94]$.

In vitro, lymphatic capillary networks have been created in collagen [93], fibrin [94], and collagen-fibrin mixtures [95], using slow interstitial flow as a guiding principle for extensive formation of interconnected tubes with open lumen. In all cases, a growth factor like VEGF or VEGF-C or a stimulant like PMA is also necessary for initiating lymphatic growth, via proliferation and migration [96], and this has been achieved for example using an engineered version of $\mathrm{VEGF}_{121}$ with a transglutaminase tag that covalently binds it into a fibrin matrix upon crosslinking with Factor XIII [94,97], as well as a similar variant of VEGF-C (unpublished). $\mathrm{VEGF}_{165}$ naturally binds to sulfated proteoglycans in the extracellular matrix, and it is believed that this facilitates gradient formation to guide angiogenic sprouts through cell-released proteolysis; however, it has also been suggested that the matrix-binding properties of VEGF allows interstitial flow to act as an organizational cue because flow skews cell-released proteases in the flow direction and therefore matrix-liberated VEGF becomes biased, with an amplified gradient, in the flow direction [94,98]. Thus, both growth cues (growth factors) and biophysical cues (interstitial flow) are required for functional lymphatic capillary morphogenesis in vitro.

In vivo, VEGF-C delivery is being investigated as a potential therapy for lymphedema and in wound healing. For example, following again the idea of heparin-binding growth factors in nature, a VEGF-C/VEGF heparin-binding domain fusion protein was engineered and expressed in vivo via adenovirus, leading to more efficient lymphangiogenesis than the WTVEGF-C delivered the same way [99]. In vivo models of lymphedema [100,101] have been used to show promise in the potential of VEGF-C therapy [102]; however, it is not 
known whether improvements in experimental lymphedema in such models are due to lymphangiogenesis, improved lymphatic drainage due to VEGF-C effects on lymphatic function such as downstream lymphatic pump function [103], or other effects. It is important to note that simply upregulating VEGF-C in a tissue with normal lymphatic vasculature can drive lymphatic hyperplasia and dysfunction [104], and thus more research, aimed at delivering VEGF-C in more physiologically relevant ways (spatially and temporally controlled and co-delivery with other important growth factors) and at evaluating and optimizing function as well as morphology, is needed before therapeutic lymphangiogenesis becomes a clinical reality.

\section{Conclusions}

Expanding knowledge of lymphatic function and immune cell trafficking into lymphatic vessels has allowed new insights into the mechanisms by which antigens from the peripheral tissues are efficiently delivered to DCs residing in the lymph nodes and likewise by which DCs from the periphery home there. Moreover, this understanding has guided new strategies in vaccine delivery, designing biomaterial delivery systems with physical characteristics that enable facile transport through the tissue interstitium under the influence of this interstitial flow to target immature DCs that reside in the lymph nodes. Moreover, such biomaterials systems can serve as powerful tools to more precisely study specific responses of, and complex interactions between, immune cells (this is the subject of another chapter in this issue). For example, dendritic cell maturation following exposure to polymer films and particles has been characterized [105,106]; materials systems have been designed to explore the connections of innate and adaptive immunity in DC activation [83]; surfaces have been designed to quantitatively and controllably mimic DC-T cell interactions [84]; and biomaterial microparticles have been loaded with chemoattractants to investigate the effects of spatial gradients on cell migration [107]. These and other advances, including continued development of engineered lymph node mimics and lymphatic vessels, designed in view of the physiology and biology of the immune system in mind, will be useful both as experimental tools to probe biological mechanisms and as therapeutic tools in immunomodulation.

\section{References}

[1] Angeli V, Ginhoux F, Llodra J, Quemeneur L, Frenette PS, Skobe M, et al. B cell-driven lymphangiogenesis in inflamed lymph nodes enhances dendritic cell mobilization. Immunity 2006;24(2):203-15.

[2] Steinman RM, Inaba K. Myeloid dendritic cells. J Leukoc Biol 1999;66:205-8.

[3] Leak LV. Structure of lymphatic capillaries in lymph formation. Fed Proc 1976;35(8):1863-71.

[4] Leak LV. Permeability of lymphatic capillaries. J Cell Biol 1971;50(2):300-23.

[5] Spiegel M, Vesti B, Shore A, Franzeck UK, Becker F, Bollinger A. Pressure of lymphatic capillaries in human skin. Am J Phys 1992;262(4): H1208-10.

[6] Fischer M, Franzeck UK, Herrig I, Costanzo U, Wen S, Schiesser M, et al. Flow velocity of single lymphatic capillaries in human skin. Am J Phys 1996;39(1):H358-63.
[7] Swartz MA, Berk DA, Jain RK. Transport in lymphatic capillaries. 1. Macroscopic measurements using residence time distribution theory. Am J Phys 1996;39(1):H324-9.

[8] Schmid-Schonbein GW. Microlymphatics and lymph flow. Physiol Rev 1990;70(4):987-1028.

[9] Trzewik J, Mallipattu SK, Artmann GM, Delano FA, Schmid-Schonbein GW. Evidence for a second valve system in lymphatics: endothelial microvalves. FASEB J 2001;15:1711-7.

[10] Quick CM, Venugopal AM, Gashev AA, Zawieja DC, Stewart RH. Intrinsic pump-conduit behavior of lymphangions. Am J Phys 2007;292(4):R1510-8.

[11] von der Weid PY, Zawieja DC. Lymphatic smooth muscle: the motor unit of lymph drainage. Int J Biochem Cell Biol 2004;36(7):1147-53.

[12] Zawieja DC, Davis KL, Schuster R, Hinds WM, Granger HJ. Distribution, propagation, and coordination of contractile activity in lymphatics. Am J Phys 1993;264(4):H1283-91.

[13] Kaldjian EP, Gretz JE, Anderson AO, Shi YH, Shaw S. Spatial and molecular organization of lymph node $\mathrm{T}$ cell cortex: a labyrinthine cavity bounded by an epithelium-like monolayer of fibroblastic reticular cells anchored to basement membrane-like extracellular matrix. Int Immunol 2001;13(10):1243-53.

[14] Bajenoff M, Egen JG, Koo LY, Laugier JP, Brau F, Glaichenhaus N, et al. Stromal cell networks regulate lymphocyte entry, migration, and territoriality in lymph nodes. Immunity 2006;25(6):989-1001.

[15] Sixt M, Kanazawa N, Seig M, Samson T, Roos G, Reinhardt DP, et al. The conduit system transports soluble antigens from the afferent lymph to resident dendritic cells in the $\mathrm{T}$ cell area of the lymph node. Immunity 2005;22(1):19-29.

[16] Cyster JG. Chemokines, sphingosine-1-phosphate, and cell migration in secondary lymphoid organs. Ann Rev Immunol 2005;23:127-59.

[17] Luther SA, Tang HL, Hyman PL, Farr AG, Cyster JG. Coexpression of the chemokines ELC and SLC by T zone stromal cells and deletion of the ELC gene in the plt/plt mouse. Proc Nat Acad Sci U S A 2000;97(23): 12694-9.

[18] Cyster JG. Chemokines and the homing of dendritic cells to the T cell areas of lymphoid organs. J Exp Med 1999;189:447-50.

[19] Cyster JG. Chemokines-chemokines and cell migration in secondary lymphoid organs. Science 1999;286(5447):2098-102.

[20] Luther SA, Bidgol A, Hargreaves DC, Schmidt A, Xu Y, Paniyadi J, et al. Differing activities of homeostatic chemokines CCL19, CCL21, and CXCL12 in lymphocyte and dendritic cell recruitment and lymphoid neogenesis. J Immunol 2002;169(1):424-33.

[21] Bousso P, Robey E. Dynamics of CD8+ T cell priming by dendritic cells in intact lymph nodes. Nat Immunol 2003;4:579-85.

[22] Brand CU, Hunziker T, Braathen LR. Isolation of human skin-derived lymph: flow and output of cells following sodium lauryl sulphate-induced contact dermatitis. Arch Dermatol Res 1992;284(3):123-6.

[23] Drake RE, Gabel JC. Effect of outflow pressure on intestinal lymph flow in unanesthetized sheep. Am J Physiol 1991;260(4 Pt 2):668-71.

[24] Bajenoff M, Egen JG, Qi H, Huang AY, Castellino F, Germain RN. Highways, byways and breadcrumbs: directing lymphocyte traffic in the lymph node. Trends Immunol 2007;28(8):346-52.

[25] Hirakawa S, Brown LF, Kodama S, Paavonen K, Alitalo K, Detmar M. VEGF-C-induced lymphangiogenesis in sentinel lymph nodes promotes tumor metastasis to distant sites. Blood 2007;109(3):1010-7.

[26] Rockson SG. Lymphedema. Am J Med 2001;110(4):288-95.

[27] Ohl L, Mohaupt M, Czeloth N, Hintzen G, Kiafard Z, Zwirner J, et al. CCR7 governs skin dendritic cell migration under inflammatory and steady-state conditions. Immunity 2004;21(2):279-88.

[28] Ritter U, Wiede F, Mielenz D, Kiafard Z, Zwirner J, Korner H. Analysis of the CCR7 expression on murine bone marrow-derived and spleen dendritic cells. J Leukoc Biol 2004;76(2):472-6.

[29] Sozzani S, Allavena P, D'Amico G, Luini W, Bianchi G, Kataura M, et al. Differential regulation of chemokine receptors during dendritic cell maturation: a model for their trafficking properties. J Immunol 1998;161(3):1083-6.

[30] Saeki H, Moore AM, Brown MJ, Hwang ST. Cutting edge: secondary lymphoid-tissue chemokine (SLC) and CC chemokine receptor 7 (CCR7) 
participate in the emigration pathway of mature dendritic cells from the skin to regional lymph nodes. J Immunol 1999;162(5):2472-5.

[31] Gunn MD, Kyuwa S, Tam C, Kakiuchi T, Matsuzawa A, Williams LT, et al. Mice lacking expression of secondary lymphoid organ chemokine have defects in lymphocyte homing and dendritic cell localization. J Exp Med 1999;189(3):451-60.

[32] Robbiani DF, Finch RA, Jager D, Muller WA, Sartorelli AC, Randolph GJ. The leukotriene C-4 transporter MRP1 regulates CCL19 (MIP-3 beta, ELC)-dependent mobilization of dendritic cells to lymph nodes. Cell 2000;103(5):757-68.

[33] Chary SR, Jain RK. Direct measurement of interstitial convection and diffusion of albumin in normal and neoplastic tissues by fluorescence photobleaching. Proc Natl Acad Sci U S A 1989;86:5385-9.

[34] Patel DD, Koopmann W, Imai T, Whichard LP, Yoshie O, Krangel MS. Chemokines have diverse abilities to form solid phase gradients. Clin Immunol 2001;99(1):43-52.

[35] Boardman KC, Swartz MA. Interstitial flow as a guide for lymphangiogenesis. Circ Res 2003;92:801-8.

[36] Benoit JN. Effects of f-Met-Leu-Phe-induced inflammation on intestinal lymph flow and lymphatic pump behavior. Am J Physiol 1992;262(2 25-2):G199-202.

[37] Granger DN, Zimmerman BJ, Sekizuka E, Grisham MB. Intestinal microvascular exchange in the rat during luminal perfusion with formylmethionyl-leucyl-phenylalanine. Gastroenterology 1988;94(3):673-81.

[38] Koizumi T, Kubo K, Hirai K, Shinozaki S, Kobayashi T, Sekiguchi M, et al. Effects of continuous infusion of nitroglycerin on pulmonary hemodynamics, lung lymph balance, and prostanoid products in the response to endotoxin in awake sheep. Lung 1993;171(1):19-30.

[39] Randolph GJ, Angeli V, Swartz MA. Dendritic cell trafficking to lymph nodes via lymphatic vessels. Nat Rev Immunol 2005;5:1-12.

[40] Johnson LA, Clasper S, Holt AP, Lalor PF, Baban D, Jackson DG. An inflammation-induced mechanism for leukocyte transmigration across lymphatic vessel endothelium. J Exp Med 2006;203(12):2763-77.

[41] Kuroshima S, Sawa Y, Kawamoto T, Yamaoka Y, Notani K, Yoshida S, et al. Expression of Toll-like receptors 2 and 4 on human intestinal lymphatic vessels. Microvasc Res 2004;67(1):90-5.

[42] Wilson NS, El-Sukkari D, Belz GT, Smith CM, Steptoe RJ, Heath WR, et al. Most lymphoid organ dendritic cell types are phenotypically and functionally immature. Blood 2003;102(6):2187-94.

[43] Gatza E, Okada CY. Tumor cell lysate-pulsed dendritic cells are more effective than TCR Id protein vaccines for active immunotherapy of $\mathrm{T}$ cell lymphoma. J Immunol 2002;169(9):5227-35.

[44] Timmerman JM, Czerwinski DK, Davis TA, Hsu FJ, Benike C, Hao ZM, et al. Idiotype-pulsed dendritic cell vaccination for B-cell lymphoma: clinical and immune responses in 35 patients. Blood 2002;99(5):1517-26.

[45] Wang RF, Wang HY. Enhancement of antitumor immunity by prolonging antigen presentation on dendritic cells. Nat Biotech 2002;20(2):149-54.

[46] Harzstark AL, Small EJ. Immunotherapy for prostate cancer using antigen-loaded antigen-presenting cells: APC8015 (Provenge). Expert Opin Biol Ther 2007;7(8):1275-80.

[47] Knutson KL. Technology evaluation: DCVax, Northwest Biotherapeutics. Curr Opin Mol Ther 2002;4(4):403-7.

[48] Fifis T, Gamvrellis A, Crimeen-Irwin B, Pietersz GA, Li J, Mottram PL, et al. Size-dependent immunogenicity: therapeutic and protective properties of nano-vaccines against tumors. J Immunol 2004;173(5):3148-54.

[49] Little SR, Lynn DM, Ge Q, Anderson DG, Puram SV, Chen J, et al. Poly-beta amino ester-containing microparticles enhance the activity of nonviral genetic vaccines. Proc Natl Acad Sci U S A 2004;101(26):9534-9.

[50] Storni T, Ruedl C, Schwarz K, Schwendener RA, Renner WA, Bachmann MF. Nonmethylated CG motifs packaged into virus-like particles induce protective cytotoxic $\mathrm{T}$ cell responses in the absence of systemic side effects. J Immunol 2004;172(3):1777-85.

[51] Wang C, Ge Q, Ting D, Nguyen D, Shen HR, Chen JZ, et al. Molecularly engineered poly(ortho ester) microspheres for enhanced delivery of DNA vaccines. Nat Mater 2004;3(3):190-6.

[52] Bonifaz LC, Bonnyay DP, Charalambous A, Darguste DI, Fujii S, Soares $\mathrm{H}$, et al. In vivo targeting of antigens to maturing dendritic cells via the DEC-205 receptor improves T cell vaccination. J Exp Med 2004;199(6):815-24.

[53] Trumpfheller C, Finke JS, Lopez CB, Moran TM, Moltedo B, Soares $\mathrm{H}$, et al. Intensified and protective $\mathrm{CD} 4+\mathrm{T}$ cell immunity in mice with anti-dendritic cell HIV gag fusion antibody vaccine. J Exp Med 2006;203(3):607-17.

[54] Bozzacco L, Trumpfheller C, Siegal FP, Mehandru S, Markowitz M, Carrington M, et al. DEC-205 receptor on dendritic cells mediates presentation of HIV gag protein to CD8+ T cells in a spectrum of human MHC I haplotypes. Proc Natl Acad Sci U S A 2007;104(4):1289-94.

[55] Kwon YJ, James E, Shastri N, Frechet JM. In vivo targeting of dendritic cells for activation of cellular immunity using vaccine carriers based on pH-responsive microparticles. Proc Natl Acad Sci U S A 2005;102(51):18264-8.

[56] van Broekhoven CL, Parish CR, Demangel C, Britton WJ, Altin JG. Targeting dendritic cells with antigen-containing liposomes: a highly effective procedure for induction of antitumor immunity and for tumor immunotherapy. Cancer Res 2004;64(12):4357-65.

[57] Swartz MA, Fleury ME. Interstitial flow and its effects in soft tissues. Ann Rev Biomed Eng 2007;9:229-56.

[58] Rutkowski JM, Swartz MA. A driving force for change: interstitial flow as a morphoregulator. Trends Cell Biol 2007;17(1):44-50.

[59] Reddy ST, Berk DA, Jain RK, Swartz MA. A sensitive in vivo model for quantifying interstitial convective transport of injected macromolecules and nanoparticles. J Appl Physiol 2006;101:1162-9.

[60] Reddy ST, Rehor A, Schmoekel HG, Hubbell JA, Swartz MA. In vivo targeting of dendritic cells in lymph nodes with poly(propylene sulfide) nanoparticles. J Cont Rel 2006;112(1):26-34.

[61] Oussoren C, Storm G. Liposomes to target the lymphatics by subcutaneous administration. Adv Drug Deliv Rev 2001;50:143-56.

[62] Aukland K, Reed RK. Interstitial-lymphatic mechanisms in the control of extracellular fluid volume. Phys Rev 1993;73(1):1-78.

[63] Nishioka Y, Yoshino H. Lymphatic targeting with nanoparticulate system. Adv Drug Deliv Rev 2001;47(1):55-64.

[64] Hunter RL. Overview of vaccine adjuvants: present and future. Vaccine 2002;20(Suppl. 3):S7-12.

[65] Salerno-Goncalves R, Sztein MB. Cell-mediated immunity and the challenges for vaccine development. Trends Microbiol 2006;14(12): 536-42.

[66] Ulevitch RJ. Therapeutics targeting the innate immune system. Nat Rev Immunol 2004;4(7):512-20.

[67] Akira S, Takeda K. Toll-like receptor signalling. Nat Rev Immunol 2004;4(7):499-511.

[68] Beutler B. Inferences, questions and possibilities in Toll-like receptor signalling. Nature 2004;430(6996):257-63.

[69] Mata-Haro V, Cekic C, Martin M, Chilton PM, Casella CR, Mitchell TC. The vaccine adjuvant monophosphoryl lipid A as a TRIF-biased agonist of TLR4. Science 2007;316(5831):1628-32.

[70] Hemmi H, Takeuchi O, Kawai T, Kaisho T, Sato S, Sanjo H, et al. A Tolllike receptor recognizes bacterial DNA. Nature 2000;408(6813):740-5.

[71] Alexopoulou L, Holt AC, Medzhitov R, Flavell RA. Recognition of double-stranded RNA and activation of NF-kappaB by Toll-like receptor 3. Nature 2001;413(6857):732-8.

[72] Vernacchio L, Bernstein H, Pelton S, Allen C, MacDonald K, Dunn J, et al. Effect of monophosphoryl lipid A (MPL) on T-helper cells when administered as an adjuvant with pneumocococcal-CRM197 conjugate vaccine in healthy toddlers. Vaccine 2002;20(31-32):3658-67.

[73] Little SR, Lynn DM, Puram SV, Langer R. Formulation and characterization of poly (beta amino ester) microparticles for genetic vaccine delivery. J Cont Rel 2005;107(3):449-62.

[74] Kwon YJ, Standley SM, Goh SL, Frechet JM. Enhanced antigen presentation and immunostimulation of dendritic cells using acid-degradable cationic nanoparticles. J Cont Rel 2005;105(3):199-212.

[75] Dempsey PW, Allison ME, Akkaraju S, Goodnow CC, Fearon DT. C3d of complement as a molecular adjuvant: bridging innate and acquired immunity. Science 1996;271(5247):348-50.

[76] Nielsen CH, Fischer EM, Leslie RGQ. The role of complement in the acquired immune response. Immunology 2000;100(1):4-12. 
[77] Kopf M, Abel B, Gallimore A, Carroll M, Bachmann MF. Complement component $\mathrm{C} 3$ promotes T-cell priming and lung migration to control acute influenza virus infection. Nat Med 2002;8(4):373-8.

[78] Kemper C, Atkinson JP. T-cell regulation: with complements from innate immunity. Nat Rev Immunol 2007;7(1):9-18.

[79] Gorbet MB, Sefton MV. Biomaterial-associated thrombosis: roles of coagulation factors, complement, platelets and leukocytes. Biomaterials 2004;25(26):5681-703.

[80] Nilsson B, Ekdahl KN, Mollnes TE, Lambris JD. The role of complement in biomaterial-induced inflammation. Mol Immunol 2007;44(1-3):82-94.

[81] Andersson J, Bexborn F, Klinth J, Nilsson B, Ekdahl KN. Surfaceattached PEO in the form of activated Pluronic with immobilized factor $\mathrm{H}$ reduces both coagulation and complement activation in a whole-blood model. J Biomed Mater Res A 2006;76(1):25-34.

[82] Gorbet MB, Sefton MV. Complement inhibition reduces material-induced leukocyte activation with PEG modified polystyrene beads (Tentagel) but not polystyrene beads. J Biomed Mater Res A 2005;74(4):511-22.

[83] Reddy ST, van der Vlies AJ, Simeoni E, Angeli V, Randolph GJ, O’Neil $\mathrm{CP}$, et al. Exploiting lymphatic transport and complement activation in nanoparticle vaccines. Nat Biotechnol 2007;25(10):1159-64.

[84] Doh J, Irvine DJ. Immunological synapse arrays: patterned protein surfaces that modulate immunological synapse structure formation in $\mathrm{T}$ cells. Proc Natl Acad Sci U S A 2006;103(15):5700-5.

[85] Irvine DJ, Doh J. Synthetic surfaces as artificial antigen presenting cells in the study of $\mathrm{T}$ cell receptor triggering and immunological synapse formation. Semin Immunol 2007;19(4):245-54.

[86] Stachowiak AN, Irvine DJ. Inverse opal hydrogel-collagen composite scaffolds as a supportive microenvironment for immune cell migration. J Biomed Mater Res A 2007, epub ahead of print.

[87] Suematsu S, Watanabe T. Generation of a synthetic lymphoid tissue-like organoid in mice. Nat Biotechnol 2004;22:1539-45.

[88] Okamoto N, Chihara R, Shimizu C, Nishimoto S, Watanabe T. Artificial lymph nodes induce potent secondary immune responses in naive and immunodeficient mice. J Clin Invest 2007;117:997-1007.

[89] Adams RH, Alitalo K. Molecular regulation of angiogenesis and lymphangiogenesis. Nat Rev Mol Cell Biol 2007;8(6):464-78.

[90] Liersch R, Detmar M. Lymphangiogenesis in development and disease. Thromb Haemost 2007;98(2):304-10.

[91] Rutkowski JM, Boardman KC, Swartz MA. Characterization of lymphangiogenesis in a model of adult skin regeneration. Am J Physiol Heart Circ Physiol 2006;291:H1402-10.

[92] Goldman J, Conley KA, Raehl A, Bondy DM, Pytowski B, Swartz MA, et al. Regulation of lymphatic capillary regeneration by interstitial flow in skin. Am J Physiol Heart Circ Physiol 2007;292(5):H2176-83.

[93] Ng CP, Helm CE, Swartz MA. Interstitial flow differentially stimulates blood and lymphatic endothelial cell morphogenesis in vitro. Microvasc Res 2004;68(3):258-64
[94] Helm CE, Fleury ME, Zisch AH, Boschetti F, Swartz MA. Synergy between interstitial flow and VEGF directs capillary morphogenesis in vitro through a gradient amplification mechanism. Proc Natl Acad Sci U S A 2007;44:15779-84.

[95] Helm CE, Zisch AH, Swartz MA. Engineered blood and lymphatic capillaries in 3D VEGF-fibrin-collagen matrices with interstitial flow. Biotech Bioeng 2007;96(1):167-76.

[96] Goldman J, Rutkowski JM, Shields JD, Pasquier MC, Cui Y, Schmoekel HG, et al. Cooperative and redundant roles of VEGFR-3 and VEGFR-2 signaling in adult lymphangiogenesis. FASEB J 2007;21(4): 1003-12.

[97] Ehrbar M, Djonov VG, Schnell C, Tschanz SA, Martiny-Baron G, Schenk U, et al. Cell-demanded liberation of VEGF121 from fibrin implants induces local and controlled blood vessel growth. Circ Res 2004;94(8):1124-32.

[98] Fleury ME, Boardman KC, Swartz MA. Autologous morphogen gradients by subtle interstitial flow and matrix interactions. Biophys $\mathrm{J}$ 2006;91:113-21.

[99] Tammela T, He Y, Lyytikkä J, Jeltsch M, Markkanen J, Pajusola K, et al. Distinct architecture of lymphatic vessels induced by chimeric vascular endothelial growth factor-C/vascular endothelial growth factor heparinbinding domain fusion proteins. Circ Res 2007;100:1468-75.

[100] Slavin SA, van den Abbeele AD, Losken A, Swartz MA, Jain RK. Return of lymphatic function after flap transfer for acute lymphedema. Ann Surg 1999;229:421-7.

[101] Rutkowski JM, Moya M, Johannes J, Goldman J, Swartz MA. Secondary lymphedema in the mouse tail: lymphatic hyperplasia, VEGF-C upregulation, and the protective role of MMP-9. Microvasc Res 2006;72: $161-71$.

[102] Cheung L, Han J, Beilhack A, Joshi S, Wilburn P, Dua A, et al. An experimental model for the study of lymphedema and its response to therapeutic lymphangiogenesis. Bio Drugs 2006;20(6):363-70.

[103] Breslin JW, Gaudreault N, Watson KD, Reynoso R, Yuan SY, Wu MH Vascular endothelial growth factor-C stimulates the lymphatic pump by a VEGF receptor-3-dependent mechanism. Am J Physiol Heart Circ Physiol 2007;293(1):H709-18.

[104] Goldman J, Le TX, Skobe M, Swartz MA. Overexpression of VEGF-C causes transient lymphatic hyperplasia but not increased lymphangiogenesis in regenerating skin. Circ Res 2005;96:1193-9.

[105] Yoshida M, Babensee JE. Poly(lactic-co-glycolic acid) enhances maturation of human monocyte-derived dendritic cells. J Biomed Mater Res A 2004;71(1):45-54.

[106] Bennewitz NL, Babensee JE. The effect of the physical form of poly(lactic-co-glycolic acid) carriers on the humoral immune response to co-delivered antigen. Biomaterials 2005;26(16):2991-9.

[107] Zhao X, Jain S, Larman BH, Gonzalez S, Irvine DJ. Directed cell migration via chemoattractants released from degradable microspheres. Biomaterials 2005;26(24):5048-63. 